\title{
NeuroImage
}

ELSEVIER

www.elsevier.com/locate/ynimg

NeuroImage 32 (2006) 956 - 967

\section{Neural correlates of artificial syntactic structure classification}

\author{
Christian Forkstam, ${ }^{\text {a,b }}$ Peter Hagoort, ${ }^{\text {a }}$ Guillen Fernandez, ${ }^{\text {a }}$ \\ Martin Ingvar, ${ }^{\mathrm{b}}$ and Karl Magnus Petersson ${ }^{\mathrm{a}, \mathrm{b}, \mathrm{c}, *}$ \\ ${ }^{a}$ F.C. Donders Centre for Cognitive Neuroimaging, Radboud University Nijmegen, The Netherlands \\ ${ }^{\mathrm{b}}$ Stockholm Brain Institute, Cognitive Neurophysiology Research Group, Karolinska Institutet, Stockholm, Sweden \\ ${ }^{\mathrm{c}}$ CSI, Centre for Intelligent Systems, Universidade do Algarve, Faro, Portugal
}

Received 12 June 2005; revised 13 March 2006; accepted 20 March 2006

Available online 6 June 2006

\begin{abstract}
The human brain supports acquisition mechanisms that extract structural regularities implicitly from experience without the induction of an explicit model. It has been argued that the capacity to generalize to new input is based on the acquisition of abstract representations, which reflect underlying structural regularities in the input ensemble. In this study, we explored the outcome of this acquisition mechanism, and to this end, we investigated the neural correlates of artificial syntactic classification using event-related functional magnetic resonance imaging. The participants engaged once a day during an 8-day period in a short-term memory acquisition task in which consonantstrings generated from an artificial grammar were presented in a sequential fashion without performance feedback. They performed reliably above chance on the grammaticality classification tasks on days 1 and 8 which correlated with a corticostriatal processing network, including frontal, cingulate, inferior parietal, and middle occipital/occipitotemporal regions as well as the caudate nucleus. Part of the left inferior frontal region (BA 45) was specifically related to syntactic violations and showed no sensitivity to local substring familiarity. In addition, the head of the caudate nucleus correlated positively with syntactic correctness on day 8 but not day 1, suggesting that this region contributes to an increase in cognitive processing fluency.

(C) 2006 Elsevier Inc. All rights reserved.
\end{abstract}

Keywords: Artificial grammar learning; Functional neuroimaging; fMRI; Inferior frontal cortex; Basal ganglia

\footnotetext{
* Corresponding author. F.C. Donders Centre for Cognitive Neuroimaging, PO Box 9101, 6500 HB Nijmegen, The Netherlands. Fax: +31 243610652.

E-mail address: karl.magnus.petersson@fcdonders.ru.nl (K.M. Petersson)

Available online on ScienceDirect (www.sciencedirect.com).
}

\section{Introduction}

Humans are equipped with acquisition mechanisms that extract structural regularities implicitly from experience without the induction of an explicit model (Reber, 1967; Stadler and Frensch, 1998). This capacity was explored in the seminal work of Reber (1967), indicating that humans can successfully classify strings generated from an implicitly acquired artificial grammar. This suggests that relevant structural information can be acquired from environmental input, and Reber (1967) proposed that this process is intrinsic to natural language learning. Following this suggestion, it has recently been argued that artificial grammar learning (AGL) is a relevant model for investigating aspects of language learning in infants (Gomez and Gerken, 2000), exploring differences between human and animal learning relevant to the narrow faculty of language (Hauser et al., 2002), and language learning in adults (Friederici et al., 2002; Petersson et al., 2004). A complementary perspective on AGL views this as a model for investigating implicit learning (Forkstam and Petersson, 2005b; Seger, 1994; Stadler and Frensch, 1998). Reber (1967) defined implicit learning as the process by which an individual comes to respond appropriately to the statistical structure of the input ensemble. More recently, Seger (1994), following Reber, suggested four defining characteristics of implicit learning: (1) limited explicit access to the knowledge acquired; subjects typically cannot provide a sufficient explicit account of what they have learnt; (2) the nature of the knowledge acquired is more complex than simple associations or simple exemplar-specific frequency counts; (3) implicit learning does not involve explicit hypothesis testing but is an automatic (incidental) consequence of the type and amount of processing performed on the stimuli; (4) implicit learning does not rely on declarative memory mechanisms that engages the MTL memory system. Furthermore, Reber (1967) suggested that humans can acquire implicit knowledge of the underlying structure through an inductive statistical learning process, and that the acquired knowledge is put to use during grammaticality classification. At the time, Reber (1967) argued that implicit learning mechanisms abstracted 'rule-based' knowledge but 
has since modified his position (Reber, 1993), and more recent studies seem to suggest that dual mechanisms might be at play (Meulemans and Van der Linden, 1997).

Support for the implicit character of artificial grammar learning also comes from lesion studies on amnesic patients. Knowlton and Squire (1996) investigated amnesic patients and normal controls on the original AGL task as well as a transfer version of the task, where the capacity to remap the underlying structure onto a new alphabet (i.e., a new set of letters) was investigated. The patients and their normal controls performed similarly on both AGL versions, while the amnesic patients could not explicitly retrieve either complete string or 2 and 3 letter substring information. Knowlton and Squire (1996) argued that AGL depends on implicit acquisition of both abstract and exemplar-specific information, the latter indicated by the acquisition of distributional information of local sequential regularities (i.e., substring or chunk regularities\}. They also argued for the existence of abstract (e.g., 'rule-based') representations based on the results from the transfer version of AGL. In addition, the acquisition of long-distance dependencies, as opposed to local substring dependencies, has been demonstrated in visuomotor sequence learning and in AGL (Poletiek, 2002). This suggests that some form of abstraction of grammatical structure takes place during acquisition, and, for example, Meulemans and Van der Linden (1997) have argued that the classification capacity cannot be explained entirely in terms of the acquisition of local sequential regularities. Moreover, it is known that infants can learn and generalize over local sequential regularities and several studies have shown rapid (on the order of 2-10 min) 'rule-abstraction' (Marcus et al., 1999), and AGL (Gomez and Gerken, 1999), as well as acquisition of transition probabilities in artificial syllable sequences (Saffran et al., 1996). In the study of Gomez and Gerken (1999), infants also demonstrated some transfer capacity, suggesting that they were abstracting beyond the transition probabilities holding between particular items in the grammar.

The artificial grammar used by Reber (1967), here and subsequently referred to as the Reber grammar, is an example of a right-linear phrase structure grammar which generates a regular language. The Reber grammar, like any right-linear phrase structure grammar, can be implemented in a finite-state architecture (Fig. 1; Petersson, 2004), and we used this grammar in the present study to generate the stimulus material. The finite-state machine can be

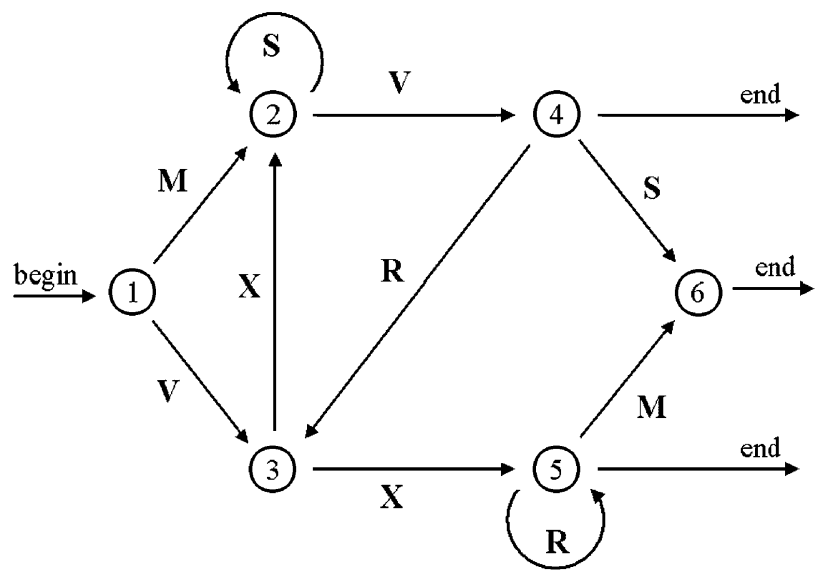

Fig. 1. Transition graph representation of the Reber machine used in the study. The transition graph representation of the Reber machine which was used to generate the grammatical strings in the present study (cf., Reber and Allen, 1978). viewed as an explicit generating mechanism or a recognition device for a formal language (e.g., Davis et al., 1994). In general, a formal (artificial) grammar serves as an intentional definition (Chomsky, 1986) of a language and represents a formal specification of the mechanism that generates structural regularities in the output. It should be noted that the finite-state architecture is not limited to capture local substring dependencies but also incorporates longdistance dependencies (as long as there is a fixed finite upper bound for these dependencies; for further discussion of this issue, see Petersson, 2004).

A number of functional magnetic resonance imaging (fMRI) studies have investigated implicit (e.g., Lieberman et al., 2004; Petersson et al., 2004; Seger et al., 2000; Skosnik et al., 2002) and explicit learning of material generated from artificial grammars (e.g., Fletcher et al., 1999; Strange et al., 2001), as well as natural (Musso et al., 2003) or artificial languages (e.g., Opitz and Friederici, 2003). In the explicit learning studies, the experimental task can be characterized as explicit problem solving with performance feedback (Petersson et al., 2004). In this set-up, the participants are explicitly instructed to try to extract the underlying rules based on feedback ('trial-and-error search'). To overcome the explicit nature of the acquisition task in these experiments, and with the perspective that AGL might serve as a model for language acquisition, Petersson et al. (2004) investigated a group of participants on a grammaticality classification task, using an implicit acquisition paradigm without feedback in which the participants were only exposed to positive examples (i.e., wellformed strings) generated from the Reber grammar. The results showed that artificial syntactic violations activated Broca's region (left Brodmann's area (BA) 44/45). In the present study, we further investigated the role of the left inferior frontal region (BA 44/45) in artificial syntactic classifications, using the experimental setup of Petersson et al. (2004) with some modifications: we used a balanced associative chunk strength (ACS) design (cf., e.g., Knowlton and Squire, 1996; Lieberman et al., 2004; Meulemans and Van der Linden, 1997), we included several acquisition sessions over 8 days, and the strings were presented in a sequential fashion, one letter at a time, which requires temporal integration of information. The balanced ACS design, in which the factors grammaticality status (grammatical/non-grammatical) and ACS (high/low) were independently controlled in a $2 \times 2$ factorial design, allowed us to investigate the neural correlates of classification based on the acquisition of abstract regularities independent of classification based on local substring familiarity. It has been argued that sensitivity to the level of ACS is a reflection of a statistical fragment-based learning mechanism, while sensitivity to grammaticality status, independent of ACS, is related to a structure-based acquisition mechanism. Moreover, it is likely that learning based on ACS reflects explicit declarative memory mechanism involving the medial temporal lobe (Lieberman et al., 2004), while implicit learning of grammaticality status independent of ACS reflects a procedural learning mechanism involving the basal ganglia. These kind of dissociated regional specific learning effects were thus expected in the current study. Specifically, this line of reasoning predicts an increased sensitivity to ACS in the MTL, while grammaticality related effects should correlate with the basal ganglia. This would be in line with a recent suggestion concerning the relative contribution of the declarative and procedural memory systems in language (see, e.g., Ullman, 2004). Finally, in the present study, as in the classical AGL paradigm, there were both acquisition and classification sessions; 
during each acquisition session, participants were engaged in a short-term memory task without performance feedback using an acquisition sample generated from the Reber grammar. Subsequent to the acquisition sessions on day 1 and day 8 , the subjects were asked to classify new items, not previously encountered, as grammatical or non-grammatical, guided by their immediate intuitive impression (i.e., to guess based on 'gut feeling'). Typically, the subjects performed reliably above chance. With these modifications, we attempted to replicate our previous finding that the left inferior frontal cortex (BA 44/45) is specifically sensitive to artificial syntactic violations.

\section{Materials and methods}

\section{Participants}

Fourteen healthy right-handed Dutch speaking participants with an university background volunteered to participate in the study (the inclusion criterion of a classification performance above $60 \%$ correct trials by test day 8 excluded 2 subjects from further fMRI data analysis; 12 participants ( 8 females), mean age $\pm \mathrm{SD}=23 \pm 3$ years). All subjects were pre-screened, and none of the subjects used any medication, had a history of drug abuse, head trauma, neurological or psychiatric illness, or a family history of neurological or psychiatric illness. Written informed consent was obtained according to the Declaration of Helsinki, and the local medical ethics committee approved the study.

\section{Stimulus material}

We generated 569 grammatical $(G)$ strings from the Reber grammar (5-12 consonants from the alphabet $\{\mathrm{M}, \mathrm{S}, \mathrm{V}, \mathrm{R}, \mathrm{X}\}$; see Fig. 1). For each item, we calculated frequency distribution of 2 and 3 letter chunks for both terminal and complete string positions in order to derive the associative chunk strength (ACS) for each item (cf., Knowlton and Squire, 1996; Meulemans and Van der Linden, 1997). Then iteratively, we randomly selected 100 strings, generating an acquisition set which were comparable in terms of 2 and 3 letter chunks to the complete string set. Subsequently, we generated the non-grammatical (NG) string, derived from each remaining $G$ string by a switch of letters in two non-terminal positions, and these were selected to match the $\mathrm{G}$ strings in terms of both terminal and complete string ACS (i.e., collapsed over order information within strings). Finally, in an iterative procedure, we randomly selected 2 sets of 56 strings each from the remaining $\mathrm{G}$ strings, in order to generate the 2 classification sets consisting of $50 \% \mathrm{G}$ and NG strings, as well as 50\% high and low ACS strings relative to ACS information in the acquisition set and independent of grammaticality status. Thus, the stimulus material included an acquisition set and two classification sets (all sets were pair-wise disjoint). The classification sets were used for the $2 \times 2$ factorial design of the classification task. Thus, each classification set consisted of 28 strings of each string type: high ACS grammatical (HG), low ACS grammatical (LG), high ACS non-grammatical (HNG), and low ACS non-grammatical (LNG).

\section{Experimental procedure}

The complete experiment included 8 days with an acquisition session each day, followed on the 1 st and the 8 th days by a classification test during which EPI-BOLD data were acquired with fMRI. During both acquisition and classification sessions, each string was centrally presented letter-by-letter on a computer screen (2.7-6.9 s corresponding to 5-12 letters; 300-ms letter presentation duration, 300-ms inter-letter-interval) using the Presentation software (http://nbs.neuro-bs.com). During acquisition, all subjects were presented with the 100 acquisition strings, randomly ordered for each acquisition session. When the last letter in a string disappeared, the subject was instructed to immediately reconstruct the string from memory by typing on a keyboard in a self-paced fashion. The subjects were not provided with any sort of performance feedback, and only positive examples (i.e., grammatical strings) were presented during acquisition. During classification on days 1 and 8, the participants were presented with novel letter strings, in a similar way as during acquisition, while fMRI measurements were conducted. The subjects were informed about the grammaticality classification task before the first acquisition session and instructed to classify the novel strings as grammatical or not in a yes/no forced choice procedure, by means of their immediate intuitive impression (i.e., guessing based on 'gut feeling'). To minimize eye movements, two white horizontal bars were presented centrally on a black background throughout the scanning sessions. After a 1-s pre-stimulus period, the strings were presented between these bars, followed by a 1-s delay and 2.5-s fixation cross period, indicating that the subject had to respond by pressing either left or right index finger, balanced within subject over sessions. The classification sets and string presentation order were balanced over subjects. A sensorimotor decision control task was included as a low-level baseline condition. In this task, strings of repeated letters $\mathrm{P}$ or $\mathrm{L}$ (matched in string length to the classification set) were randomly interspersed and presented in the same fashion as the classification strings and subjects had to respond by pressing the right or left index finger, respectively. The stimuli were presented via an LCD projector standing outside the scanner room, projecting the computer display onto a semitransparent screen that the subject comfortably viewed through a mirror device mounted on the head-coil.

\section{MRI data acquisition}

During the classification tasks, we acquired whole head T2*weighted EPI-BOLD fMRI data with a SIEMENS Trio 3-T MRscanner using an ascending slice acquisition sequence (volume $\mathrm{TR}=$ $2.8 \mathrm{~s}, \mathrm{TE}=40 \mathrm{~ms}, 90^{\circ}$ flip-angle, 35 axial slices, slice-matrix size $=$ $64 \times 64$, slice thickness $=3 \mathrm{~mm}$, slice gap $=0.5 \mathrm{~mm}, \mathrm{FOV}=22.4$ $\mathrm{mm}$, isotropic voxel size $=3.5 \times 3.5 \times 3.5 \mathrm{~mm}^{3}$ ). For the structural MR image volume, a high-resolution T1-weighted 3D MPRAGE sequence was used (volume TR $=1960 \mathrm{~ms}, \mathrm{TE}=4.43 \mathrm{~ms}, 8^{\circ} \mathrm{flip}$ angle, 176 coronal slices, slice-matrix size $=256 \times 208$, slice thickness $=1.0 \mathrm{~mm}$, voxel-size $=1 \mathrm{~mm} \times 1 \mathrm{~mm} \times 1 \mathrm{~mm}$ ).

\section{MRI data analysis}

We used the SPM2 shareware (http://www.fil.ion.ucl.ac.uk/ $\mathrm{spm}$ ) for image pre-processing and statistical analysis. Due to field inhomogeneities, we corrected the structural T1 image for any linear trend in the bias field (Ashburner, 2002). To exclude nonbrain information in the estimation of the normalization parameters, we skin stripped both the structural image of each subject and the MNI 152 T1 template by means of an inclusive mask with subject segmented grey and white matter components. The 
functional EPI-BOLD images were realigned, slice-time corrected, and the subject-mean functional MR images were co-registered with the corresponding structural MR images. These were subsequently spatially normalized (i.e., the normalization transformations were generated from the structural MR images and applied to the functional MR images), and the functional EPIBOLD images were transformed into an approximate Talairach space (Talairach and Tournoux, 1988) as defined by the SPM template and spatially filtered with an isotropic 3D spatial Gaussian kernel (FWHM $=10 \mathrm{~mm}$ ). The fMRI data were proportionally scaled to account for global effects and analyzed statistically using the general linear model and statistical parametric mapping (Friston et al., 1995) in a two-step mixed design procedure. At the first level, single-subject fixed effect analyses were conducted. The linear model included mini-block regressors to model the string presentation period of the HG, HNG, LG, LNG, and the control items, separated on correct and incorrect responses as well as test day. We temporally convolved these explanatory variables with the canonical hemodynamic response function provided by SPM2. We included the realignment parameters for movement artefact correction and a temporal high-pass filter to account for various low-frequency effects as effects of no interest. For the second-level analysis, we generated single-subject contrast images for the correctly classified HG, HNG, LG, LNG and control items relative to the implicit baseline and used these in a one-way subject-separated random effects repeated measures ANOVA (including the following factors: condition [HG day $1 / 8, \mathrm{HNG}$ day $1 / 8$, LG day $1 / 8$, LNG day $1 / 8$, control day $1 / 8$ ] and subject) with non-sphericity correction. Because of high performance on test day 8 , we did not further analyze the few incorrectly classified trials. The normalized T1 grey matter component of each subject was used as an inclusive mask in the statistical analysis.

Statistical inference was based on the cluster-size statistics from the relevant second-level SPM[F] and SPM[T] volumes. Clusters at a significance level of $P<0.05$ corrected for multiple nonindependent comparisons based on the family-wise error are listed in the corresponding tables. Local maxima within significant clusters were subsequently described at a significance level of $P<$ 0.05 corrected for multiple non-independent comparisons based on false discovery rate (FDR, Genovese et al., 2002). In this way, $\mathrm{SPM}[\mathrm{F}]$ volumes were generated to investigate effects related to grammatical vs. sensorimotor classification tests (i.e., effects of event type: HG, HNG, LG, LNG vs. control; correct responses only, within subjects, tested across subjects; Fig. 4a, left; Table 2) and effects related to grammaticality and ACS manipulations, for both within and across test days (i.e., effects of grammatical status (grammatical/non-grammatical), ACS status (high/low), or interaction within or between test days within subjects, tested across subjects; Fig. 4a, right; Table 3, left). To disentangle the effects observed in the latter omnibus test, a region of interest analysis was performed on regions centered on the detected local maxima (5$\mathrm{mm}$ radius, Table 3 , right). We also included regions within the caudate nucleus and the hippocampus bilaterally derived from a similar study (Lieberman et al., 2004) in the analysis. Finally, we also generated SPM[T] volumes thresholded at $Z=3.39$ ( $P=$ 0.0005 uncorrected) and applied small volume correction to the significant regions of interest defined by the SPM[F] volume (Table 4; for the grammaticality manipulation see Fig. 4b). In the following, we use the terms activation and deactivation as synonyms for a relative increase and decrease in BOLD signal, respectively. For reasons of portability of the results, we used the
Talairach nomenclature (Talairach and Tournoux, 1988) in the tables with the original SPM coordinates. Finally, the behavioral data were analyzed with a repeated measures ANOVA (including the following factors: grammaticality status [grammatical/nongrammatical], ACS status [high/low], and test day [1/8]) using the statistics package R (http://www.r-project.org). The dependent measure in this analysis was the endorsement rate (i.e., \% strings perceived as grammatical).

\section{Results}

\section{Behavioral results}

The analysis of the classification performance showed that the subjects were sensitive to both grammaticality (GRAM) and ACS. The participants showed additional sensitivity to grammaticality on day 8 as compared to day 1, while they showed no additional sensitivity to ACS between test days. Consistent with previous findings, the overall correct classification performance ( $50 \%$ correct expected by chance) was clearly above chance on both test occasions (test day 1 : mean $=62 \%$ correct; range $=49$ $79 ; T(11)=5.1, P=0.0002$; test day $8:$ mean $=87 \%$ correct, range $=75-96, T(11)=18, P<0.0001)$. Thus, subjects classified the items reliably above chance on the first day, and the classification performance improved with repeated acquisition sessions. We then analyzed the performance data in terms of endorsement rate (i.e., strings accepted as grammatical, cf., Meulemans and Van der Linden, 1997). The classification results in terms of endorsement rate showed that subjects were sensitivity to both grammaticality status and ACS (Table 1, Figs. 2 and 3: mean (standard deviation) test day 1, HG: 75(9)\%; LG: 57(13)\%; HNG: 48(15)\%; LNG: 34(12)\%; test day 8: HG: 98(2)\%; LG: 79(7)\%; HNG: 19(18)\%; LNG: 9(12)\%). A repeated measure ANOVA with test day (1/8), grammaticality (G/NG) and $\operatorname{ACS}(\mathrm{H} / \mathrm{L})$ as within factors, showed significant main effects of grammaticality $(F(11,84)=467, P<0.0001)$ and ACS $(F(11,84)=41, P<0.0001)$, while the main effect of test day was non-significant $(F(11,84)=1.0, P=0.32)$. However, there was a significant interaction between test day and grammaticality $(F(11,84)=117, P<0.0001)$, while the interaction of test day and ACS was non-significant $(F(11,84)=0.12, P=0.72)$. This suggests that the factor grammaticality is the main contributor to the increased classification performance between the first and last test day, suggesting that subjects developed a significant sensitivity to grammatical status during the 8 days of acquisition. No other interactions reached significance $($ GRAM $\times$ ACS: $F(11,84)=1.8, P=0.19$; TEST $\times$ GRAM $\times$ ACS: $F(11,84)=$ $0.38, P=0.54)$. In order to investigate the potential effect repeated letters within strings and whether subjects showed a

Table 1

Endorsement rates over grammaticality and ACS factor level categories

\begin{tabular}{|c|c|c|c|c|}
\hline & \multicolumn{2}{|l|}{ Test day 1} & \multicolumn{2}{|l|}{ Test day 8} \\
\hline & High ACS & Low ACS & High ACS & Low ACS \\
\hline Grammatical & (9) & (13) & (2) & (7) \\
\hline Non-grammatical & (15) & (12) & (18) & (12) \\
\hline
\end{tabular}

Percentage of items endorsed (i.e., perceived as grammatical) by condition (grammatical/non-grammatical $\times$ high/low associative chunk strength (ACS) status; mean performance level and standard deviation). 


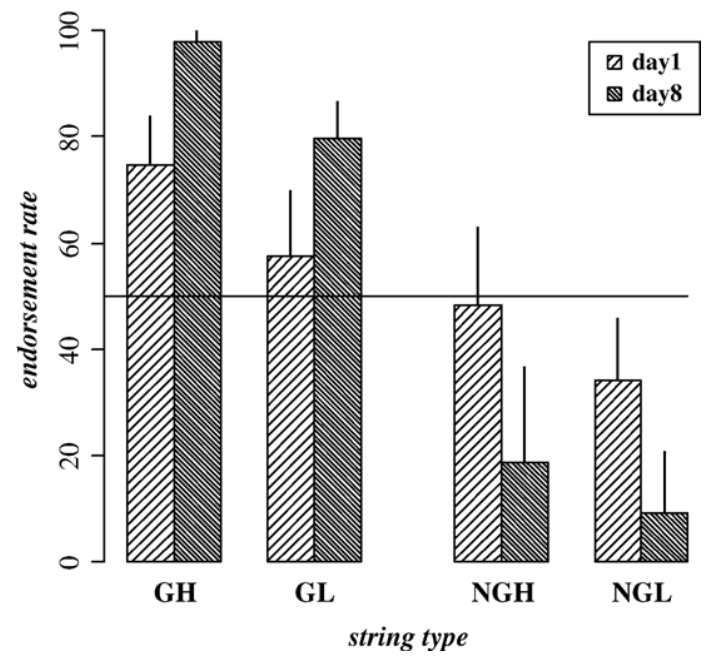

Fig. 2. Endorsement rates over grammaticality and ACS factor level categories. The endorsement rates (i.e., string perceived as grammatical) as a function of grammaticality status as well as associative chunk strength (ACS; $\mathrm{HG}=$ grammatical high ACS strings, $\mathrm{LG}=$ grammatical low ACS strings, $\mathrm{HNG}=$ non-grammatical high $\mathrm{ACS}$ strings, $\mathrm{LNG}=$ nongrammatical low ACS strings). The endorsement rate of grammatical vs. non-grammatical items increases as a function of repeated acquisition for both high and low ACS strings. Error bars correspond to one standard deviation.

classification bias to the presence of repeating letters within strings, we analyzed the classification performance (endorsement rate) with a repeated measure ANOVA including the factor 'presence of repeating letters'. The results showed no effect of letter repetitions and the subjects still showed a significant effect of both grammaticality status and ACS (GRAM: $F(7,216)=467$, $P<0.0001$; ACS: $F(7,216)=66.6, P<0.0001)$, while no interactions reached significance (all interaction terms: $F(7,216)<$ 2, $P>0.15)$.

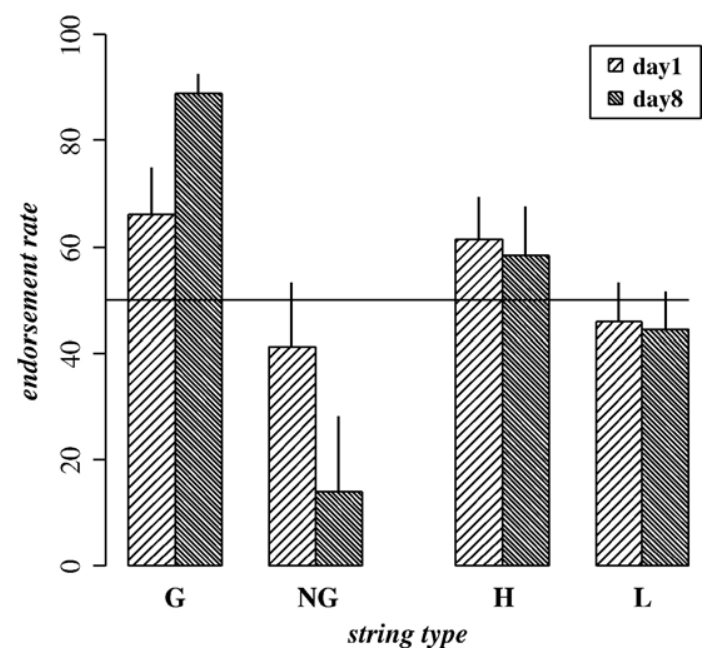

Fig. 3. Endorsement rates over grammaticality and ACS main factor categories. The endorsement rates (i.e., string perceived as grammatical) as a function of grammaticality status $(\mathrm{G}=$ grammatical strings, $\mathrm{NG}=$ nongrammatical strings) as well as associative chunk strength (ACS; $\mathrm{H}=$ high ACS strings, $\mathrm{L}=$ low ACS strings). The endorsement rate for grammatical vs. non-grammatical items, but not for high vs. low ACS items, increases as a function of repeated acquisition sessions. Error bars correspond to one standard deviation.
On the test day 1 , we observed significant main effects of grammaticality $(F(11,36)=49.2, P<0.001)$ and $\operatorname{ACS}(F(11,36)=$ 19.5, $P=0.0001)$. This was also the case for test day 8 (grammaticality: $F(11,36)=680, P<0.001$; ACS: $F(11,36)=$

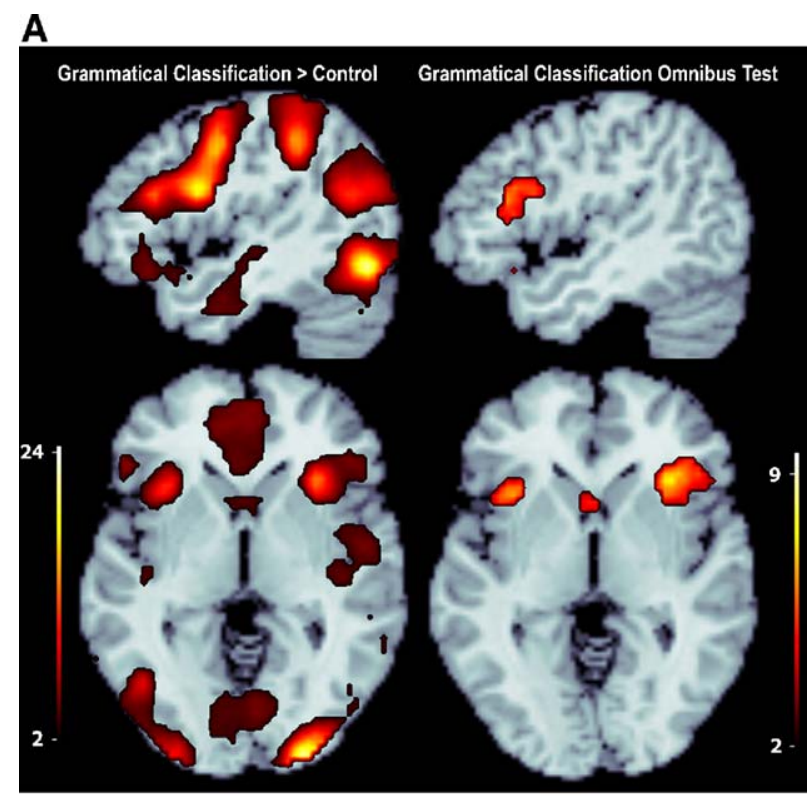

B

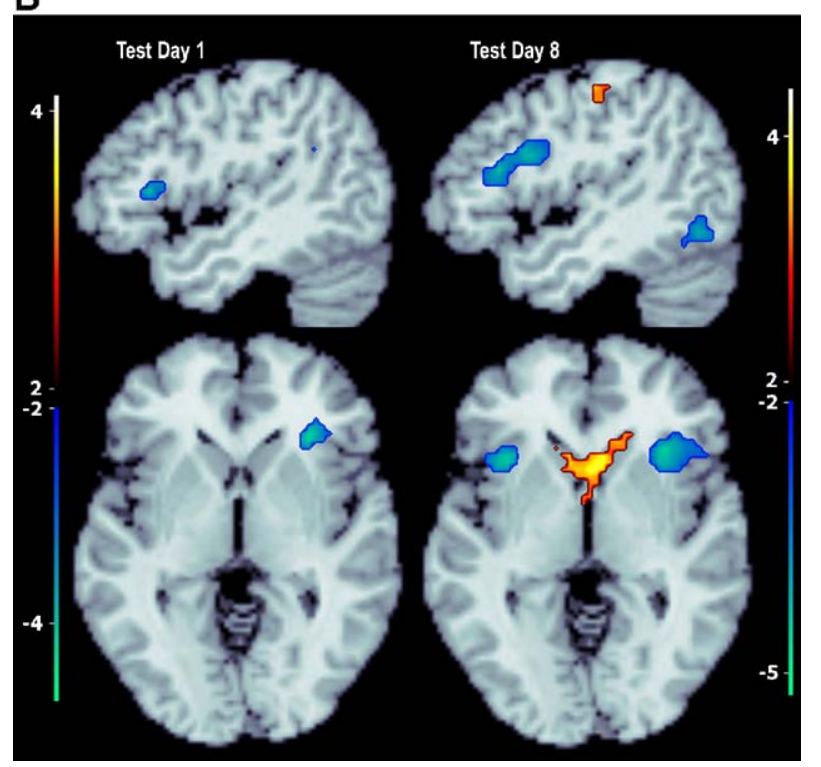

Fig. 4. (A) Regions significantly sensitive to grammatical classification (left) and omnibus ANOVA test (right). Left: the neural correlates of grammatical classification compared to the sensorimotor baseline condition during both test days. Right (Omnibus test): the regions where grammaticality, associative chunk strength, test day, or any interaction thereof explained a significant amount of the observed variability. Threshold at $P=0.05$ corrected for multiple non-independent comparisons using the false discovery rate. Activations are projected onto the normalized structural T1 image from a single subject $(x=-45 ; z=-3$; left is left; upper slices are in the left hemisphere). (B) Regions significantly sensitive to grammatical status (days 1 and 8 ). The regions significantly sensitive to grammaticality status (grammatical $>$ non- grammatical items in red, non-grammatical $>$ grammatical items in blue; only correct response trials included). Left: test day 1. Right: test day 8 . Threshold and projection template: see panel (A) $(x=-45 ; z=-3$; left is left; upper slices are in the left hemisphere). 
23.9, $P<0.001)$. The interaction between grammaticality and ACS was not significant on either test day. Moreover, the subjects showed a stable $d$-prime effect in discriminating between grammatical and non-grammatical strings on both test days (mean $d$-prime values: day $1=0.66$; range $=0-1.7$; day $8=2.3$; range $=$ $1.5-3.7)$ as well as a significant increase in $d$-prime over test days (paired $t$ test: $T=5.6, P<0.0005)$. No significant response bias was found (mean beta-values: day $1=0.96$; range $=0.8-1.1$; day $8=1.0 ;$ range $=0.4-2.5$ )

We further investigated the effects of grammaticality, following Chang and Knowlton (2004; see also Lieberman et al., 2004) who argued that ACS is not a useful cue for the low ACS items but that correct performance has to be based on knowledge of structural regularities rather than local substring knowledge. Similarly to Lieberman et al. (2004), we observed significant effects for grammaticality for both high and low ACS strings on both test days (test day 1: HG > HNG: $F(11,12)=24, P=0.0005 ; \mathrm{LG}>$
LNG: $F(11,12)=17, P=0.0016$; test day 8: HG $>$ HNG: $F(11$, $12)=239, P<0.0001$; LG $>$ LNG: $F(11,12)=348, P<0.0001)$. We also observed significant effect of ACS for both grammatical and non-grammatical strings on both test days (test day 1: $\mathrm{HG}>$ LG: $F(11,12)=21, P=0.0008$; HNG $>$ LNG: $F(11,12)=17, P=$ 0.002; test day 8: HG $>$ LG: $F(11,12)=71, P<0.0001$; HNG $>$ LNG: $F(11,12)=9.7, P=0.001)$. In addition, we compared LG vs. HNG based on the argument that this contrast maximally tax structural vs. substring knowledge; if grammaticality status is used for classification, the acceptance of an LG item would crucially depend on the grammaticality status of the item, while if substring knowledge is used, the low ACS status would promote a rejection decision. In contrast, if substring knowledge is used for classification, the acceptance of HNG items would depend on the high ACS status, while if grammaticality status is used, the grammaticality status would indicate a rejection decision. We found a preference for LG over HNG strings already on test day 1 (LG >

Table 2

Regional BOLD effect induced by grammaticality classification

\begin{tabular}{|c|c|c|c|c|c|c|c|}
\hline Region (Brodmann's area) & Cluster $P$ value & $\mathrm{cm}^{3}$ & Voxel $T_{99}$ value & Voxel $P$ value & $x$ & $y$ & $z$ \\
\hline \multicolumn{8}{|c|}{ Left middle/inferior frontal and precentral region } \\
\hline 4 & $<0.001$ & 42.1 & 26.5 & $<0.001$ & -51 & -3 & 45 \\
\hline $6 / 44$ & & & 24.2 & $<0.001$ & -48 & 3 & 24 \\
\hline 45 & & & 17.2 & $<0.001$ & -45 & 27 & 21 \\
\hline \multicolumn{8}{|c|}{ Left frontal operculum/anterior insula } \\
\hline 47 & $<0.001$ & 3.8 & 15.6 & $<0.001$ & -30 & 21 & 0 \\
\hline \multicolumn{8}{|c|}{ Right middle/inferior frontal, frontal operculum/anterior insula, and precentral region } \\
\hline 6 & $<0.001$ & 48.8 & 21.7 & $<0.001$ & 48 & 9 & 27 \\
\hline $45 / 46$ & & & 18.6 & $<0.001$ & 45 & 30 & 21 \\
\hline 47 & & & 18.4 & $<0.001$ & 33 & 24 & 0 \\
\hline \multicolumn{8}{|c|}{ Anterior cingulate/medial frontal region } \\
\hline 32 & $<0.001$ & 17.9 & 18.1 & $<0.001$ & -3 & 3 & 57 \\
\hline 32 & & & 17.2 & $<0.001$ & 9 & 15 & 48 \\
\hline 8 & & & 17.0 & $<0.001$ & -9 & 12 & 48 \\
\hline \multicolumn{8}{|c|}{ Left inferior/superior parietal region } \\
\hline 7 & $<0.001$ & 33.7 & 23.4 & $<0.001$ & -24 & -63 & 51 \\
\hline 40 & & & 20.1 & $<0.001$ & -42 & -42 & 42 \\
\hline 40 & & & 11.7 & $<0.001$ & -48 & -42 & 21 \\
\hline \multicolumn{8}{|c|}{ Right inferior/superior parietal region } \\
\hline 7 & $<0.001$ & 34.7 & 21.9 & $<0.001$ & 30 & -57 & 51 \\
\hline $7 / 39$ & & & 21.7 & $<0.001$ & 30 & -66 & 36 \\
\hline 40 & & & 15.5 & $<0.001$ & 48 & -33 & 48 \\
\hline \multicolumn{8}{|c|}{ Right superior temporal region } \\
\hline $22 / 21$ & $<0.001$ & 3.5 & 15.6 & $<0.001$ & 51 & -42 & 12 \\
\hline \multicolumn{8}{|c|}{ Left middle/inferior occipital and fusiform/inferior temporal region } \\
\hline $19 / 37$ & $<0.001$ & 25.5 & 26.9 & $<0.001$ & -45 & -69 & -9 \\
\hline 18 & & & 22.2 & $<0.001$ & -30 & -93 & -6 \\
\hline \multicolumn{8}{|c|}{ Right middle/inferior occipital, fusiform/inferior temporal region } \\
\hline 18 & $<0.001$ & 32.9 & 27.7 & $<0.001$ & 30 & -93 & -3 \\
\hline $19 / 37$ & & & 25.9 & $<0.001$ & 48 & -63 & -12 \\
\hline Right cerebellum & & & 15.9 & $<0.001$ & 30 & -63 & -30 \\
\hline
\end{tabular}

Regions more active during grammaticality classification compared to sensorimotor control classification (cf., Fig. 4a left). Cluster threshold at a significance level of $P<0.05$ corrected for multiple non-independent comparisons using the family-wise error rate are reported together with local maxima within these clusters thresholded at a significance level of $P<0.05$ corrected for multiple non-independent comparisons using the false discovery rate $\left(T_{99}\right.$ value $=T$ value for 99 degrees of freedom; $x, y, z=$ the original SPM $x, y, z$ coordinates in millimeter of the MNI space). 
HNG; $T(11)=1.5, P=0.075)$, which was significant on day $8(T(11)=10.8, P<0.0001)$. Taken together, these results suggest that grammaticality status independent of ACS is used for structural generalization in classifying novel strings and provide support for the notion that grammatical structure other than the fragment features is used for classification.

\section{fMRI results}

Compared to the sensorimotor decision baseline, the grammaticality classification activated a similar set of regions during both days 1 and 8 (Fig. 4a, left, Table 2). These results were highly similar to our recent study (Petersson et al., 2004). These activations included the inferior frontal regions bilaterally, centered on BA 44/45, extending anteriorly into BA 47, including the frontal operculum/anterior insula (BA 13/15/47), and posteriorly into the anterior precentral BA $4 / 6$. Additional prefrontal activations included the medial frontal and anterior cingulate cortex (BA $32 / 8$ ) bilaterally, extending laterally into anterior middle and superior frontal regions (BA 6/8/9/10). Bilateral posterior activations included the inferior parietal cortex (BA 39/40, extending into the inferior parts of $\mathrm{BA} 7$ ), the precuneus, and the posterior cingulate cortex (BA 7/31) bilaterally. Bilateral occipital activations were centered on the middle and inferior occipital regions (BA 18/19) and extended into the lateral fusiform cortex (BA 19/ 37) and right cerebellum. Additionally, we observed bilateral temporal activations in the anterior superior (BA 22) and middle (BA 21) temporal regions. The only regions that differed significantly between day 1 and day 8 and showed greater activity during day 8 compared to day 1 was a left anterior superior temporal region (BA 38; cluster $P=0.05$, corrected, with local maximum at $[x, y, z]=[-36,9,-24], Z=4.1, P=0.01$, FDR corrected), and an occipital region centered on lingual gyrus extending anteriorly along the gyrus and superiorly into cuneus (BA 17/18/19; cluster $P<0.001$, corrected; $[x, y, z]=[-18,-69$, 9], $Z=4.7, P=0.01$, FDR corrected).

In a subset of this set of regions, we found that a significant amount of the variability were explained by grammaticality, ACS, test day and their interactions (SPM[F] ANOVA on correct trials only; see Fig. 4a, right and Table 3, left). This subset spanned over regions including the lateral prefrontal cortices bilaterally, centered on the inferior frontal region (BA 45/47), extending into middle frontal cortex (BA 46) and frontal operculum/anterior insula (BA 13/15/47). It further included regions in the anterior (BA 24/32) and posterior cingulate cortex (BA 23/31), the right inferior parietal (BA 39), and the superior temporal (BA 22) regions, as well as the head of the caudate nucleus, bilaterally.

To elaborate further on which experimental manipulations that carried explanatory value, we performed a regions-of-interest analysis on the cluster local maxima (Table 3, right). We observed significant effects of non-grammatical vs. grammatical strings (both days 1 and 8) in the left (BA 44/45) and right (BA 45/46)

Table 3

Omnibus ANOVA test-regional BOLD effect jointly induced by the experimental manipulations

\begin{tabular}{|c|c|c|c|c|c|c|c|c|c|c|c|}
\hline \multirow[t]{2}{*}{ Region } & \multirow[t]{2}{*}{ BA } & \multirow[t]{2}{*}{$\mathrm{cm}^{3}$} & \multirow[t]{2}{*}{$P$ (FDR) } & \multirow[t]{2}{*}{$Z^{99}$} & \multirow[t]{2}{*}{$x$} & \multirow[t]{2}{*}{$y$} & \multirow[t]{2}{*}{$z$} & \multicolumn{2}{|l|}{$\mathrm{ACS}$} & \multicolumn{2}{|l|}{$\underline{\text { GRAM }}$} \\
\hline & & & & & & & & d1 & $\mathrm{d} 8$ & d1 & $\mathrm{d} 8$ \\
\hline \multirow[t]{4}{*}{ Left IFC } & \multirow[t]{4}{*}{45} & \multirow[t]{6}{*}{5.1} & 0.007 & 4.2 & -45 & 24 & 18 & & & $\mathrm{NG}>\mathrm{G}$ & $\mathrm{NG}>\mathrm{G}$ \\
\hline & & & 0.007 & 4.3 & -42 & 18 & 24 & & & $\mathrm{NG}>\mathrm{G}$ & $\mathrm{NG}>\mathrm{G}$ \\
\hline & & & 0.012 & 4.0 & -42 & 21 & 12 & & & $\mathrm{NG}>\mathrm{G}$ & $\mathrm{NG}>\mathrm{G}$ \\
\hline & & & 0.014 & 3.9 & -45 & 12 & 21 & & & $\mathrm{NG}>\mathrm{G}$ & $\mathrm{NG}>\mathrm{G}^{\dagger}$ \\
\hline \multirow[t]{2}{*}{ Left IFC/FOP } & 47 & & 0.003 & 4.5 & -36 & 18 & 3 & $\mathrm{~L}>\mathrm{H}$ & & & $\mathrm{NG}>\mathrm{G}$ \\
\hline & 47 & & 0.005 & 4.3 & -33 & 18 & -9 & $\mathrm{~L}>\mathrm{H}$ & & $\mathrm{NG}>\mathrm{G}$ & $\mathrm{NG}>\mathrm{G}$ \\
\hline \multirow[t]{5}{*}{ Right IFC } & 46 & \multirow[t]{5}{*}{11.2} & 0.008 & 4.2 & 45 & 27 & 21 & $\mathrm{~L}>\mathrm{H}$ & $\mathrm{L}>\mathrm{H}$ & $\mathrm{NG}>\mathrm{G}$ & $\mathrm{NG}>\mathrm{G}$ \\
\hline & \multirow[t]{4}{*}{$45 / 46$} & & 0.008 & 4.2 & 51 & 18 & 27 & $\mathrm{~L}>\mathrm{H}$ & & $\mathrm{NG}>\mathrm{G}$ & $\mathrm{NG}>\mathrm{G}$ \\
\hline & & & 0.009 & 4.1 & 48 & 27 & 6 & $\mathrm{~L}>\mathrm{H}$ & $\mathrm{L}>\mathrm{H}$ & $\mathrm{NG}>\mathrm{G}$ & $\mathrm{NG}>\mathrm{G}$ \\
\hline & & & 0.009 & 4.1 & 48 & 21 & 24 & $\mathrm{~L}>\mathrm{H}$ & & $\mathrm{NG}>\mathrm{G}$ & $\mathrm{NG}>\mathrm{G}$ \\
\hline & & & 0.034 & 3.3 & 45 & 18 & 42 & $\mathrm{~L}>\mathrm{H}$ & & $\mathrm{NG}>\mathrm{G}$ & $\mathrm{NG}>\mathrm{G}$ \\
\hline Right IFC/FOP & 47 & & 0.000 & 5.4 & 33 & 21 & -6 & $\mathrm{~L}>\mathrm{H}$ & & $\mathrm{NG}>\mathrm{G}$ & $\mathrm{NG}>\mathrm{G}$ \\
\hline \multirow[t]{3}{*}{ Right ACC } & 32 & \multirow[t]{3}{*}{2.7} & 0.003 & 4.5 & 6 & 27 & 33 & $\mathrm{~L}>\mathrm{H}$ & & $\mathrm{NG}>\mathrm{G}$ & $\mathrm{NG}>\mathrm{G}$ \\
\hline & $24 / 32$ & & 0.008 & 4.2 & 9 & 21 & 39 & $\mathrm{~L}>\mathrm{H}$ & & $\mathrm{NG}>\mathrm{G}$ & $\mathrm{NG}>\mathrm{G}$ \\
\hline & 24 & & 0.043 & 3.2 & 12 & 27 & 24 & $\mathrm{~L}>\mathrm{H}$ & & $\mathrm{NG}>\mathrm{G}$ & \\
\hline Right IPC & 39 & 0.6 & 0.025 & 3.5 & 54 & -51 & 33 & & & $\mathrm{NG}>\mathrm{G}$ & $\dagger$ \\
\hline Right STC & 22 & 0.4 & 0.028 & 3.4 & 51 & -48 & 18 & & $\mathrm{~L}>\mathrm{H}$ & $\mathrm{NG}>\mathrm{G}$ & $\mathrm{NG}>\mathrm{G}$ \\
\hline Left PCC & $23 / 31$ & 0.4 & 0.025 & 3.5 & -12 & -51 & 30 & $\mathrm{H}>\mathrm{L}$ & & & $\mathrm{G}>\mathrm{NG}$ \\
\hline \multirow{2}{*}{$\mathrm{PCC}$} & 31 & 1.9 & 0.009 & 4.1 & 0 & -30 & 45 & $\mathrm{H}>\mathrm{L}$ & & $\mathrm{G}>\mathrm{NG}$ & $\mathrm{G}>\mathrm{NG}$ \\
\hline & 31 & & 0.026 & 3.5 & -12 & -24 & 45 & $\mathrm{H}>\mathrm{L}$ & & & $\mathrm{G}>\mathrm{NG}$ \\
\hline Caudate nucleus & 0.5 & 0.017 & 3.7 & 3 & 15 & -3 & & $\mathrm{H}>\mathrm{L}$ & & $\mathrm{G}>\mathrm{NG}$ & \\
\hline Right Caudate, reported & & & & & 14 & 16 & 0 & & & & $\mathrm{G}>\mathrm{NG}$ \\
\hline Left Caudate, homolog to above & & & & & -14 & 16 & 0 & & & & $\mathrm{G}>\mathrm{NG}$ \\
\hline Right Hippocampus, reported & & & & & 28 & -30 & -2 & & $\mathrm{~L}>\mathrm{H}$ & & \\
\hline Left Hippocampus, homolog to above & & & & & -28 & -30 & -2 & $\mathrm{H}>\mathrm{L}$ & $\mathrm{L}>\mathrm{H}$ & & \\
\hline
\end{tabular}

Regions in which grammaticality (GRAM), associative chunk strength (ACS), and test day, or any interactions explained a significant amount of variability (i.e., omnibus ANOVA test; SPM[F] thresholded at $P=0.05$ corrected for multiple non-independent comparisons using the false discovery rate [FDR]; cf., Fig. 4a right). The right part of the table describes the region of interest analysis (radius $=5 \mathrm{~mm} ; P$ values are $\mathrm{FDR}$ corrected at $P=0.05$ ). $\mathrm{G}=$ grammatical; $\mathrm{NG}=$ non-grammatical; $\mathrm{H}=$ high ACS; L = low ACS; BA = Brodmann's area; IFC = inferior frontal cortex; FOP = frontal operculum; ACC = anterior cingulate cortex; PCC = posterior cingulate cortex; IPC = inferior parietal cortex; STC = superior temporal cortex; significant ACS $\times$ GRAM interactions during test day $1(\dagger)$ or $8(\$)$; reported $=$ region reported in Lieberman et al. (2004). 
inferior frontal regions, as well as in the ventral inferior frontal region (BA 47, extending into the frontal operculum/anterior insula, BA 13/15/47) bilaterally. We observed additional significant activations in the right anterior cingulate (BA 24/32) and the right posterior superior temporal (BA 22) regions, and on test day 1 also in the right inferior parietal region (BA 39). Interestingly, the right inferior frontal region (BA 45/46/47) and the anterior cingulate cortex (BA 32) were sensitive to the level of ACS on test day 1. This was also the case for the right inferior frontal regions on the test day 8 (low $>$ high ACS). In fact, the only frontal region which did not show any sensitivity to the level of ACS on either of the test days was the left inferior frontal cortex (BA 45). Furthermore, the posterior cingulate/precuneus region (BA 23/31) was more active for high compared to low ACS strings on day 1 and for grammatical compared to non-grammatical strings on test day 8 . The caudate nucleus displayed the same pattern of activity. In a separate analysis of the caudate nucleus and the hippocampus, based on the results reported by Lieberman et al. (2004), it was shown that the hippocampus was more active for low compared to high ACS strings on both test days. On test day 8, the caudate nucleus was significantly more active for grammatical compared to non-grammatical strings. The SPM[T] tests yielded similar results (Table 4).

Finally, following the analysis of the behavioral data, we tested the contrasts LG vs. HNG, and HNG vs. LG, in order to investigate regions specifically sensitive to structural knowledge and fragment knowledge, respectively (Table 4). We found the caudate nucleus (LG vs. HNG: $[x, y, z]=[3,18,-3]$ ) to be sensitive to grammaticality status, suggesting that this region is specifically involved in the processing of grammatical structure independent of

Table 4

Regional BOLD effects separately induced by any experimental manipulation

\begin{tabular}{|c|c|c|c|c|c|c|c|c|c|c|c|}
\hline Contrast & & Region & BA & Cluster $P$ & $\mathrm{~cm}^{3}$ & Voxel $P$ & $T_{99}$ & $Z_{99}$ & $x$ & $y$ & $z$ \\
\hline \multicolumn{12}{|c|}{ Regions sensitive to grammaticality status } \\
\hline \multirow[t]{6}{*}{ Test day 1} & $\mathrm{NG}>\mathrm{G}$ & L IFC & 45 & 0.037 & 0.2 & 0.001 & 4.1 & 3.9 & -45 & 24 & 9 \\
\hline & & R IFC & 45 & 0.044 & 0.1 & 0.003 & 3.5 & 3.4 & 51 & 27 & 3 \\
\hline & & $\mathrm{R}$ IFC & 47 & 0.002 & 3.1 & 0.001 & 4.4 & 4.2 & 45 & 36 & -12 \\
\hline & & $\mathrm{R} \mathrm{MFC}$ & 9 & 0.027 & 0.4 & 0.002 & 3.8 & 3.7 & 42 & 18 & 33 \\
\hline & & R IPL & 40 & 0.021 & 0.6 & 0.001 & 4.7 & 4.5 & 45 & -45 & 24 \\
\hline & & R IPL & 40 & 0.023 & 0.5 & 0.002 & 4.0 & 3.9 & 51 & -48 & 30 \\
\hline \multirow[t]{10}{*}{ Test day 8} & $\mathrm{NG}>\mathrm{G}$ & $\mathrm{L}$ IFC & 45 & 0.004 & 2.1 & $<0.001$ & 4.5 & 4.3 & -45 & 24 & 18 \\
\hline & & L IFC & 44 & & & $<0.001$ & 4.3 & 4.1 & -45 & 12 & 24 \\
\hline & & L FOP & 47 & 0.002 & 2.7 & $<0.001$ & 4.6 & 4.3 & -36 & 18 & 3 \\
\hline & & R IFC & 45 & $<0.001$ & 11.3 & $<0.001$ & 4.6 & 4.4 & 45 & 24 & 21 \\
\hline & & R IFC & 44 & & & $<0.001$ & 4.7 & 4.5 & 51 & 18 & 27 \\
\hline & & R FOP & 47 & & & $<0.001$ & 5.2 & 4.9 & 33 & 21 & -3 \\
\hline & & $\mathrm{R} A C C$ & 32 & 0.029 & 0.3 & 0.001 & 3.6 & 3.5 & 6 & 24 & 36 \\
\hline & $\mathrm{G}>\mathrm{NG}$ & Caud & $\mathrm{NC}$ & 0.016 & 0.8 & 0.002 & 4.0 & 3.9 & 3 & 18 & -3 \\
\hline & & PCC & 31 & 0.002 & 2.7 & 0.002 & 4.4 & 4.2 & -3 & -30 & 45 \\
\hline & & L PCC & 31 & 0.024 & 0.5 & 0.002 & 4.1 & 4.0 & -12 & -51 & 27 \\
\hline \multicolumn{12}{|c|}{ Regions sensitive to ACS status } \\
\hline \multirow[t]{4}{*}{ Test day 1} & $\mathrm{H}>\mathrm{L}$ & $\mathrm{L}$ PCC & 31 & 0.040 & 0.2 & 0.061 & 4.0 & 3.9 & -12 & -54 & 36 \\
\hline & $\mathrm{L}>\mathrm{H}$ & R FOP & 47 & 0.013 & 0.9 & 0.011 & 4.4 & 4.2 & 27 & 27 & -12 \\
\hline & & $\mathrm{R} A C C$ & 32 & 0.037 & 0.2 & 0.012 & 3.6 & 3.4 & 6 & 30 & 36 \\
\hline & & $\mathrm{ACC}$ & 32 & 0.037 & 0.2 & 0.013 & 3.4 & 3.3 & 3 & 27 & 39 \\
\hline Test day 8 & & & & n.s. & & & & & & & \\
\hline \multicolumn{12}{|c|}{ Regions sensitive to grammaticality status: low ACS strings only } \\
\hline \multirow[t]{2}{*}{ Test day 1} & $\mathrm{LNG}>\mathrm{LG}$ & R FOP & 47 & 0.040 & 0.2 & 0.036 & 3.7 & 3.6 & 30 & 27 & -3 \\
\hline & & R STC & 22 & 0.040 & 0.2 & 0.036 & 3.8 & 3.7 & 42 & -48 & 18 \\
\hline \multirow[t]{4}{*}{ Test day 8} & $\mathrm{LNG}>\mathrm{LG}$ & $\mathrm{R}$ IFC & 44 & 0.027 & 0.4 & 0.020 & 3.6 & 3.4 & 51 & 15 & 24 \\
\hline & & R FOP & 47 & 0.032 & 0.3 & 0.020 & 3.6 & 3.5 & 33 & 21 & -3 \\
\hline & $\mathrm{LG}>\mathrm{LNG}$ & L PCC & 31 & 0.010 & 1.2 & 0.012 & 3.7 & 3.6 & -6 & -27 & 45 \\
\hline & & L PCC & 31 & 0.037 & 0.2 & 0.012 & 3.7 & 3.6 & -12 & -51 & 27 \\
\hline
\end{tabular}

Regions sensitive to ACS status: NG strings only

Test days 1 and 8

n.s.

Regions specifically activated for ACS or grammatically status, respectively

Test day 1

\begin{tabular}{|c|c|c|c|c|c|c|c|c|c|c|c|}
\hline day 8 & $\begin{array}{l}\mathrm{HNG}>\mathrm{LG} \\
\mathrm{LG}>\mathrm{HNG}\end{array}$ & $\begin{array}{l}\text { R FOP } \\
\text { Caud }\end{array}$ & $\begin{array}{l}47 \\
\mathrm{NC}\end{array}$ & $\begin{array}{l}0.040 \\
0.023\end{array}$ & $\begin{array}{l}0.2 \\
0.5\end{array}$ & $\begin{array}{l}0.052 \\
0.004\end{array}$ & 3.5 & 3.4 & 36 & 24 & $\begin{array}{l}-6 \\
-3\end{array}$ \\
\hline
\end{tabular}

SPM[T] tests of the significant regions observed in the omnibus ANOVA SPM[F]-contrast (cf., Table 3 and Fig. 4b). Clusters at a significance level of $P<$ 0.05 corrected for multiple non-independent comparisons using the family-wise error rate are reported together with local maxima within these clusters at a significance level of $P<0.05$ corrected for multiple non-independent comparisons using the false discovery rate. MFC $=$ middle frontal cortex; IPL $=$ inferior parietal lobule; Caud = caudate nucleus; for additional abbreviations see Table 3. 
ACS. Conversely, we found the right frontal operculum (BA 47; $[x, y, z]=[36,24,-6])$ to be sensitive to ACS on test day 8 , suggesting that this region is involved in the processing of local substring knowledge.

\section{Discussion}

The primary objective of the present study was to replicate our previous finding (Petersson et al., 2004) that the left inferior frontal cortex (BA 44/45) is sensitive to artificial syntactic violations in a balanced chunk strength design and with a serial presentation of string symbols. This was indeed the case, although the activated frontal regions were more extensive and also included right homotopic regions. Importantly, the left inferior frontal region (BA 45) was the only frontal region which was selectively sensitive to grammaticality but not to the level of associative chunk strength (ACS). This lends support for the suggestion that the left inferior frontal cortex (BA 45) has a specific role in processing structural regularities (Petersson et al., 2004). This is also consistent with recent results showing that the left prefrontal cortex subserves syntactic processing independent of lexical meaning (Indefrey et al., 2001). In contrast, the right inferior frontal region was sensitive to ACS, potentially reflecting aspects of declarative retrieval processes or generic error detection processes (cf., Indefrey et al., 2001).

Additional regions that were sensitive to low ACS items on day 1 included the frontal operculum and anterior insula bilaterally, an effect that was absent on day 8, and displayed a constant or increasing sensitivity to syntactic violations over test days. Moreover, the caudate nucleus displayed an initial sensitivity for high ACS items but not to grammatical status on day 1, while it showed sensitivity to grammatical items but not to the level of ACS status on day 8 . Similarly, the posterior cingulate region altered its sensitivity over acquisition sessions from being sensitive to high ACS items but not to grammatical status on day 1 , to being sensitive to grammatical items but not to ACS status on day 8 .

When comparing grammaticality classification with the sensorimotor baseline, we observed a significantly activated network of regions including the left middle-inferior frontal region, the anterior cingulate cortex, the left inferior parietal, the bilateral middle-inferior occipital and the ventral occipitotemporal cortices. This is consistent with previous studies of implicit artificial grammar learning (Petersson et al., 2004; Seger et al., 2000; Skosnik et al., 2002). Furthermore, this shows that the inferior frontal region is actively engaged in the context of an extensive functional brain network, consistent with the most uncontroversial insight from functional neuroimaging suggesting that cognitive functions are implemented in functional networks (e.g., Ingvar and Petersson, 2000).

It has been argued that sensitivity to the level of ACS is a reflection of a statistical chunk-based learning mechanism while sensitivity to grammaticality status independent of ACS is related to a syntactic structure-based acquisition mechanism (Knowlton and Squire, 1996; Meulemans and Van der Linden, 1997). Moreover, sensitivity to ACS manipulation has been suggested to reflect declarative learning mechanisms dependent on medial temporal lobe structures (e.g., Opitz and Friederici, 2003). In addition, a recent fMRI study (Lieberman et al., 2004) using an AGL paradigm similar to the one used in the present study, suggested that the right caudate nucleus is sensitive to grammaticality status (i.e., more active for grammatical compared to non-grammatical items) as well as negatively correlated with medial temporal lobe activity. In the present study, the caudate nucleus was more active for grammatical compared to non-grammatical items, but we were unable to replicate the medial temporal lobe finding. Both in this and in our previous study (Petersson et al., 2004), we did not observe any significant grammaticality effects related to the medial temporal lobe. However, in the present study, we observe an effect of ACS in the medial temporal lobe (Table 3). Given that the brain lesion literature suggests that the medial temporal lobe is not necessary for implicit acquisition of knowledge (Forkstam and Petersson, 2005b; Gagnon et al., 2004; Knowlton and Squire, 1994; Knowlton and Squire, 1996; Seger, 1994), declarative mechanisms (i.e., the presence of an explicit task component) or the presence of performance feedback might explain the observed medial temporal lobe activations in several experiments using stimulus material generated from artificial grammars/languages or in rule learning (cf., e.g., Fletcher et al., 1999; Opitz and Friederici, 2003; Rose et al., 2004a; Rose et al., 2004b; Strange et al., 2001). For example, in a recent study of the weather prediction task, medial temporal lobe activity was associated with receiving positive feedback but not with correct classification (Seger and Cincotta, 2005), suggesting that the MTL might be involved in associative feedback prediction, perhaps based on sequence recognition at some level. However, one intriguing possibility is that the MTL might have a role in acquiring longdistance dependencies (Forkstam and Petersson, 2005b) or perhaps, in the context of the current study, other declarative memory processes related to the integration ACS over time, given the sequential nature of the our experimental design.

\section{On the possible role of the left inferior frontal region in the context of artificial grammar learning}

Several recent studies have suggested that the left inferior frontal region plays a role in several cognitive domains, including for example working memory (Baddeley, 1986, 2003; Baddeley et al., 1998), language processing (Bookheimer, 2002; Friederici, 2002; Hagoort, 2003, 2005; Kaan and Swaab, 2002), and musical syntax (Jackendoff, 2002; Lerdahl and Jackendoff, 1983; Patel, 2003). Additional evidence suggests that sequence learning depends on the left (Conway and Christiansen, 2001; Peigneux et al., 1999) and the right inferior frontal region (Doyon et al., 1996). Moreover, it appears that the human left inferior frontal region is important for learning sequences which contain hierarchical structure (Gelfand and Bookheimer, 2003; Petersson et al., 2004). This suggests that the left inferior frontal region is engaged in the processing of structural aspects of cognitive representations and might provide a neural infrastructure for structural integration (for further discussion of these issues, see Forkstam and Petersson, 2005a; Petersson et al., 2004, 2005). There are also evidence that the inferior frontal cortex is functionally subdivided (e.g., Bookheimer, 2002) which suggests some level of representational specificity as well as the possibility for interactive concurrent processing. Kaan and Swaab (2002) have argued that none of these regions are uniquely specific to linguistic syntactic processing. Thus, it is not unreasonable to suggest that syntactic processing is in fact dependent on a functional network of interacting brain regions. This picture is similar to the one proposed by Hagoort (2003, 2005), based on Vosse and Kempen (2000), in which the integration of various sources of linguistic information operates in parallel in a workspace for incremental unification of structured representation. 
Specifically, it is hypothesized that the left inferior frontal region subserves the unification space for language, one aspect of working memory.

Artificial grammar learning is commonly conceptualized in terms of either a structure-based ('rule') acquisition or a statistical ('chunk-based') learning mechanism. An alternative view that is placed somewhere between these two more common conceptualizations, re-traces a major trend in theoretical linguistics since the early 89s, so-called lexicalization (cf., Culicover and Jackendoff, 2005; Jackendoff, 2002), in which the distinction between lexical items and grammatical rules is vanishing and in effect shifts more of the grammar into the mental lexicon. In a 'lexicalized' picture of AGL, taking advantage of the fact that hierarchical structured information can be represented in terms of bracketed expressions (see, e.g., Davis et al., 1994), the acquisition of simple structured representations (e.g., $\left[s_{\mathrm{j}}, T, s_{\mathrm{k}}\right]$, where $s_{\mathrm{j}}$ and $s_{\mathrm{k}}$ are 'syntactic' features and $T$ a 'surface' feature) is akin to lexical learning, and the integration of such representations takes place in working memory during parsing (e.g., $\left[s_{\mathrm{j}}, T, s_{\mathrm{k}}\right]+\left[s_{\mathrm{p}}, R, s_{\mathrm{q}}\right] \rightarrow\left[s_{\mathrm{j}}, T-R\right.$, $\left.s_{\mathrm{q}}\right]$ if and only if $s_{\mathrm{k}}=s_{\mathrm{p}}$ ) after being activated (retrieved) by for example an input string. On-line incremental integration thus implements a recursive construction of complex representations (successive merging/unification, Petersson et al., 2004) from more primitive structures stored in long-term memory. We suggest that the latter process is dependent on general integrative mechanism supported by the left inferior frontal region. There is thus no need for a specific 'rule' acquisition mechanism because the parsing process utilizes general integration mechanisms already in place for merging or unifying structured representations. Finally, we note that the syntactic features of lexical items have acquired the functional role of control in this picture (cf., finite-state control, Minsky, 1967; Savage, 1998), which is thus distributed over a long-term memory representation ('mental lexicon') in terms of the control features that govern the integration process based on the selection information that is allowed to merge (for further discussion, see Forkstam and Petersson, 2005a; Petersson et al., 2005).

\section{The basal ganglia}

On day 1, the caudate nucleus was sensitive to ACS status but not grammaticality, while on day 8 , when the subject showed significantly better classification performance, this region was instead sensitive to grammaticality status but not to ACS. A recent study (Lieberman et al., 2004) using a similar AGL paradigm also reported that the caudate nucleus was sensitive to grammaticality. These findings are in line with a difference between processing mechanisms that retrieve linguistic structures and procedural processing mechanisms that might apply syntactic 'rules' (see, e.g., Ullman, 2004). Here, the basal ganglia support the procedural aspects of processing. In this context, it is of interest to note that the basal ganglia learning system (Packard and Knowlton, 2002) and the medial temporal lobe memory system (Squire and ZolaMorgan, 1991) might interact in complex ways, both competitively (Poldrack et al., 2001) as well as cooperatively (Voermans et al., 2004). One might speculate that the sequential nature of our current study might have resulted in more prominent effects related to the basal ganglia (Packard and Knowlton, 2002) compared to our previous study (Petersson et al., 2004), while the medial temporal lobe effects might be related to declarative memory processes as well as ACS integration over time (as noted above).
In relation to research on AGL in neuropsychological groups, both Parkinson (Reber and Squire, 1999; Smith et al., 2001; Witt et al., 2002) and Huntington patients (Knowlton et al., 1996) performed at normal levels. One possibility, since these studies did not control for letter chunk familiarity (e.g., ACS), it might be the case that information related to familiarity of letter chunks was sufficient to support normal or close to normal classification performance. However, with respect to Parkinson patients, Peigneux et al. (1999), which did control for ACS, also found normal or close to normal classification performance. However, given the sequential presentation of letter strings in the current study, it is conceivable that the basal ganglia play an important role in this particular context.

Neural systems supporting procedural learning and important for on-line governing of the parsing process are thought to depend on recurrent networks implemented in corticostriatal loops (see, e.g., Luciana, 2003; Nelson and Webb, 2003). Taken together, it can be argued that the processing of meaningless artificial grammar strings is dependent on the neural architecture for sequential learning, as well as regions implicated in the analysis of linguistic form (specifically the left BA 44/45). In support of this claim, classifications of $\mathrm{G}$ items correlated with the activation of the caudate nucleus when contrasted with NG items during test day 8 . Alternatively, the caudate involvement in the successful processing of $\mathrm{G}$ vs. NG items might be attributed to the development of automatic aspects of syntactic integration (unification). Moreover, the opposite contrast of comparing classifications of NG vs. G items correlated with activation of the left inferior frontal region. One possibility is that this results from integration (unification) difficulties during the processing of non-grammatical items, which would lead to a break down in the parsing process. In terms of laterality of the corticostriatal circuits, both the caudate and the inferior frontal region were active bilaterally during processing of grammaticality. The observed selective sensitivity to grammaticality, as opposed to ACS, in the left inferior frontal BA 45 suggests a left-lateral bias in the use of corticostriatal circuits for processing grammatical structure.

\section{Conclusion}

In this paper, we explored the outcome of an acquisition mechanism capable of extracting structural regularities from experience in an implicit fashion from positive examples alone and without any external supervision or feedback. To this end, we investigated the neural correlates of artificial syntactic classification using event-related functional magnetic resonance imaging. The participants engaged once a day during an 8-day period in a short-term memory acquisition task in which consonant-strings generated from an artificial grammar were presented in a sequential fashion without performance feedback. They performed reliably above chance on the grammaticality classification tasks on days 1 and 8 which correlated with a corticostriatal processing network, including frontal, cingulate, inferior parietal, and middle occipital/ occipitotemporal regions as well as the caudate nucleus. Part of the left inferior frontal region (BA 45) was specifically related to syntactic violations and showed no sensitivity to substring familiarity. In addition, the head of the caudate nucleus correlated positively with syntactic correctness on day 8 but not day 1 , suggesting that this region to contribute to an increase in cognitive processing fluency. 


\section{Acknowledgment}

This work was supported by the F. C. Donders Centre for Cognitive Neuroimaging, Vetenskapsrådet (8276), Hedlunds Stiftelse, and Stockholm County Council (ALF, FoUU).

\section{References}

Ashburner, J., 2002. Another MRI bias correction approach. 8th International Conference on Functional Mapping of the Human Brain, June $2-$ 6, 2002; Sendai, Japan (Paper presented at the).

Baddeley, A.D., 1986. Working Memory. Clarendon Press, Oxford.

Baddeley, A., 2003. Working memory: looking back and looking forward. Nature reviews. Neuroscience 4, 829-839.

Baddeley, A., Gathercole, S., Papagano, C., 1998. The phonological loop as a language learning device. Psychol. Rev. 105, 158-173.

Bookheimer, S., 2002. Functional MRI of language: new approaches to understanding the cortical organization of semantic processing. Annu. Rev. Neurosci. 25, 151-188.

Chang, G.Y., Knowlton, B.J., 2004. Visual feature learning in artificial grammar classification. J. Exper. Psychol., Learn., Mem., Cogn. 30, $714-722$.

Chomsky, N., 1986. Knowledge of Language. Praeger, New York.

Conway, C.M., Christiansen, M.H., 2001. Sequential learning in nonhuman primates. Trends Cogn. Sci. 5, 539-546.

Culicover, P.W., Jackendoff, R., 2005. Simpler Syntax. Oxford Univ. Press, Oxford.

Davis, M.D., Sigal, R., Weyuker, E.J., 1994. Computability, Complexity, and Languages: Fundamentals of Theoretical Computer Science. Academic Press, San Diego, CA.

Doyon, J., Owen, A.M., Petrides, M., Sziklas, V., Evans, A.C., 1996. Functional anatomy of visuomotor skill learning in human subjects examined with positron emission tomography. Eur. J. Neurosci. 8, $637-648$

Fletcher, P., Buchel, C., Josephs, O., Friston, K., Dolan, R., 1999. Learningrelated neuronal responses in prefrontal cortex studied with functional neuroimaging. Cereb. Cortex 9, 168-178.

Forkstam, C., Petersson, K.M., 2005. Syntactic classification of acquired structural regularities. Proc. Cogn. Sci. Soc., 696-701.

Forkstam, C., Petersson, K.M., 2005. Towards an explicit account of implicit learning. Curr. Opin. Neurol. 18, 435-441.

Friederici, A.D., 2002. Towards a neural basis of auditory sentence processing. Trends Cogn. Sci. 6, 78-84.

Friederici, A.D., Steinhauer, K., Pfeifer, E., 2002. Brain signatures of artificial language processing: evidence challenging the critical period hypothesis. Proc. Natl. Acad. Sci. U. S. A. 99, 529-534.

Friston, K., Holmes, A., Worsley, K., Poline, J.-B., Frith, C., Frackowiak, R., 1995. Statistical parametric maps in functional imaging: a general linear approach. Hum. Brain Mapp. 2, 189-210.

Gagnon, S., Foster, J.K., Turcotte, J., Jongenelis, S., 2004. Involvement of the hippocampus in implicit learning of supra-span sequences: the case of SJ. Cogn. Neuropsychol. 21, 867-882.

Gelfand, J.R., Bookheimer, S.Y., 2003. Dissociating neural mechanisms of temporal sequencing and processing phonemes. Neuron 38, $831-842$

Genovese, C.L., Lazar, N.A., Nichols, T., 2002. Thresholding of statistical maps in functional neuroimaging using the false discovery rate. NeuroImage 15, 870-878.

Gomez, R.L., Gerken, L., 1999. Artificial grammar learning by 1-year-olds leads to specific and abstract knowledge. Cognition 70, 109-135.

Gomez, R.L., Gerken, L., 2000. Infant artificial language learning and language acquisition. Trends Cogn. Sci. 4, 178-186.

Hagoort, P., 2003. How the brain solves the binding problem for language: a neurocomputational model of syntactic processing. NeuroImage 20, S18-S29.
Hagoort, P., 2005. On Broca, brain and binding: a new framework. Trends Cogn. Sci. 9, 416-423.

Hauser, M.D., Chomsky, N., Fitch, W.T., 2002. The faculty of language: what is it, who has it, and how did it evolve? Science 298, 1569-1579.

Indefrey, P., Hagoort, P., Herzog, H., Seitz, R.J., Brown, C.M., 2001. Syntactic processing in left prefrontal cortex is independent of lexical meaning. NeuroImage 14, 546-555.

Ingvar, M., Petersson, K.M., 2000. Functional maps-Cortical networks. In: Toga, A.W., Mazziotta, J.C. (Eds.), Brain Mapping: The Systems. Academic Press, San Diego, CA, pp. 111-140.

Jackendoff, R., 2002. Foundations of Language: Brain, Meaning, Grammar, Evolution. Oxford Univ. Press, UK.

Kaan, E., Swaab, T.Y., 2002. The brain circuitry of syntactic comprehension. Trends Cogn. Sci. 6, 350-356.

Knowlton, B.J., Squire, L.R., 1994. The information acquired during artificial grammar learning. J. Exper. Psychol., Learn., Mem., Cogn. 20, 79-91.

Knowlton, B.J., Squire, L.R., 1996. Artificial grammar learning depends on implicit acquisition of both abstract and exemplar-specific information. J. Exper. Psychol., Learn., Mem., Cogn. 22, 169-181.

Knowlton, B.J., Squire, L.R., Paulsen, J.S., Swerdlow, N.R., Swenson, M., 1996. Dissociations within nondeclarative memory in Huntington's disease. Neuropsychology 10, 538-548.

Lerdahl, F., Jackendoff, R., 1983. A Generative Theory of Tonal Music. MIT Press, Cambridge, MA.

Lieberman, M.D., Chang, G.Y., Chiao, J., Bookheimer, S.Y., Knowlton, B.J., 2004. An event-related fMRI study of artificial grammar learning in a balanced chunk strength design. J. Cogn. Neurosci. 16, $427-438$.

Luciana, M., 2003. The neural and functional development of human prefrontal cortex. In: de Haan, M., Johnson, M.H. (Eds.), The Cognitive Neuroscience of Development. Psychology Press, New York, pp. $157-179$.

Marcus, G.F., Vijayan, S., Bandi Rao, S., Vishton, P.M., 1999. Rule learning by seven-month-old infants. Science 283, 77-80.

Meulemans, T., Van der Linden, M., 1997. Associative chunk strength in artificial grammar learning. J. Exper. Psychol., Learn., Mem., Cogn. 23, $1007-1028$.

Minsky, M.L., 1967. Computation: Finite and Infinite Machines. PrenticeHall, Englewood Cliffs, NJ.

Musso, M., Moro, A., Glauche, V., Rijntjes, M., Reichenbach, J., Büchel, C., Weiller, C., 2003. Broca's area and the language instinct. Nat. Neurosci. 6, 774-781.

Nelson, C.A., Webb, S.J., 2003. A cognitive neuroscience perspective on early memory development. In: de Haan, M., Johnson, M.H. (Eds.), The Cognitive Neuroscience of Development. Psychology Press, New York, pp. $99-125$.

Opitz, B., Friederici, A.D., 2003. Interactions of the hippocampal system and the prefrontal cortex in learning language-like rules. NeuroImage 19, $1630-1737$.

Packard, M.G., Knowlton, B.J., 2002. Learning and memory functions of the basal ganglia. Annu. Rev. Neurosci. 25, 563-593.

Patel, A.D., 2003. Language, music, syntax and the brain. Nat. Neurosci. 6, $674-681$.

Peigneux, P., Meulemans, T., Van der Linden, M., Salmon, E., Petit, H., 1999. Exploration of implicit artificial grammar learning in Parkinson's disease. Acta Neurol. Belg. 99, 107-117.

Petersson, K.M., 2004. On the relevance of the neurobiological analogue of the finite state machine. Neurocomputing 65-66, 825-832.

Petersson, K.M., Forkstam, C., Ingvar, M., 2004. Artificial syntactic violations activate Broca's region. Cogn. Sci. 28, 383-407.

Petersson, K.M., Grenholm, P., Forkstam, C., 2005. Artificial grammar learning and neural networks. Proc. Cogn. Sci., 1726-1731.

Poldrack, R.A., Clark, J., Pare-Blagoev, E.J., Shohamy, D., Creso Moyano, J., Myers, C., Gluck, M.A., 2001. Interactive memory systems in the human brain. Nature 414, 546-550. 
Poletiek, F.H., 2002. Implicit learning of a recursive rule in an artificial grammar. Acta Psychol. 111, 323-335.

Reber, A.S., 1967. Implicit learning of artificial grammars. J. Verbal Learn. Verbal Behav. 5, 855-863.

Reber, A.S., 1993. Implicit Learning and Tacit Knowledge: An Essay on the Cognitive Unconscious. Oxford Univ. Press, New York.

Reber, A.S., Allen, R., 1978. Analogy and abstraction strategies in synthetic grammar learning: a functional interpretation. Cognition 6, $189-221$.

Reber, P.J., Squire, L.R., 1999. Intact learning of artificial grammars and intact category learning by patients with Parkinson's disease. Behav. Neurosci. 113, 235-242.

Rose, M., Haider, H., Weiller, C., Buchel, C., 2004a. The relevance of the nature of learned associations for the differentiation of human memory systems. Learn. Mem. 11, 145-152.

Rose, M., Haider, H., Weiller, C., Buchel, C., 2004b. The role of the medial temporal lobe structures in implicit learning: an event-related fMRI study. Neuron 36, 1221-1231.

Saffran, J.R., Aslin, R.N., Newport, E.L., 1996. Statistical learning by 8month-old infants. Science 274, 1926-1928.

Savage, J.E., 1998. Models of Computation: Exploring the Power of Computation. Addison-Wesley, Reading, MA.

Seger, C.A., 1994. Implicit learning. Psychol. Bull. 115, 163-196.

Seger, C.A., Cincotta, C.M., 2005. The roles of the caudate nucleus in human classification learning. J. Neurosci. 25, 2941-2951.

Seger, C.A., Prabhakaran, V., Poldrack, R.A., Gabrieli, J.D., 2000. Neural activity differs between explicit and implicit learning of artificial grammar strings: an fMRI study. Psychobiology 28, 283-292.
Skosnik, P.D., Mirza, F., Gitelman, D.R., Parrish, T.B., Mesulam, M.M., Reber, P.J., 2002. Neural correlates of artificial grammar learning. NeuroImage 17, 1306-1314.

Smith, J., Siegert, R.J., McDowall, J., Abernethy, D., 2001. Preserved implicit learning on both the serial reaction time task and artificial grammar in patients with Parkinson's disease. Brain Cogn. 45, 378-391.

Squire, L.R., Zola-Morgan, S., 1991. The medial temporal lobe memory system. Science 253, 1380-1386.

Stadler, M.A., Frensch, P.A. (Eds.), Handbook of Implicit Learning. Sage, Thousand Oaks, CA.

Strange, B.A., Henson, R.N.A., Friston, K.J., Dolan, R.J., 2001. Anterior prefrontal cortex mediates rule learning in humans. Cereb. Cortex 11, $1040-1046$.

Talairach, J., Tournoux, P., 1988. Co-planar Stereotaxic Atlas of the Human Brain: An Approach to Cerebral Imaging. Thieme Medical Publishers, New York.

Ullman, M.T., 2004. Contributions of memory circuits to language: the declarative/procedural model. Cognition 92, 231-270.

Voermans, N.C., Petersson, K.M., Daudey, L., Weber, B., van Spaendonck, K.P., Kremer, H.P.H., Fernández, G., 2004. Interaction between the human hippocampus and caudate nucleus during route recognition. Neuron 43, 427-435.

Vosse, T., Kempen, G., 2000. Syntactic structure assembly in human parsing: a computational model based on competitive inhibition and a lexicalist grammar. Cognition 75, 105-143.

Witt, K., Nuhsman, A., Deuschl, G., 2002. Intact artificial grammar learning in patients with cerebellar degeneration and advanced Parkinson's disease. Neuropsychologia 40, 1534-1540. 